\title{
Multisystem inflammatory syndrome in an adolescent girl presenting as atypical Kawasaki-like illness associated with coronavirus disease-19
}

\author{
Meghmala Sadhukhan ${ }^{1}$, Ranjit K. Joshi ${ }^{2}$, Prabin P. Pahi ${ }^{3}$ \\ From ${ }^{1}$ Associate Consultant, ${ }^{2}$ Consultant and Head, ${ }^{3}$ Consultant, Department of Paediatrics and Neonatology, Apollo Hospitals, Bhubaneswar, \\ Odisha, India
}

\begin{abstract}
During the current pandemic of Coronavirus disease (COVID)-19, multiple cases of a new condition in children have been reported with multisystem involvement associated with severe acute respiratory syndrome coronavirus-2, requiring intensive care admission. The World Health Organization has defined it as multisystem inflammatory syndrome in children and adolescents. Here, we report a similar case of a 15-year-old adolescent who presented with clinical features of Kawasaki-like illness along with multisystem involvement. The echocardiogram suggested the presence of coronary artery dilatation and the patient responded well to Intravenous immunoglobulin. Hence, pediatricians need to be aware of this condition in children with multisystemic involvement and these patients need to be treated as early as possible since they may deteriorate quickly.
\end{abstract}

Key words: Coronary artery dilatation, Intravenous immunoglobulin, PIMS-TS, MIS-C

$\mathrm{T}$ The incidence of COVID-19 infection in children was reported to be around $1.7 \%$ initially when the pandemic started [1]. However, the actual incidence is still not known as there are no population-based studies. Majority of children were thought to have asymptomatic, mild, or moderate illness initially [2]. During April-May 2020, clusters of cases involving children with Kawasaki-like illness associated with severe acute respiratory syndrome coronavirus (SARS-CoV)-2, with or without multisystemic involvement were reported from different parts of the world including the UK, Italy, and USA [3]. These children were found to have various combinations of features of typical/atypical Kawasaki disease, toxic shock syndrome, and macrophage activation syndrome [3].

Kawasaki's disease (KD) is a systemic vasculitis of medium size blood vessels [4]. The etiology is still controversial. However, some of the suggested infectious triggers of this disease include, Streptococcus pyogenes, Staphylococcus aureus, and Yersinia pseudotuberculosis as well as viruses such as adenovirus, enterovirus, Epstein-Barr virus, and coronavirus (New Haven coronavirus/HCoVNH) [4]. During the COVID-19 pandemic, Kawasaki-like illness has been reported with increased incidence from different parts of the world with atypical or incomplete clinical features $[5,6]$. The children with multisystem involvement and raised inflammatory markers were therefore

\section{Access this article online}

Received - 24 May 2021

Initial Review - 16 June 2021

Accepted - 12 July 2021

DOI: $10.32677 / \mathrm{IJCH} .2021 . v 08.108 .2978$ classified as Pediatric Inflammatory Multisystem Syndrome (PIMS) or Multisystem Inflammatory Syndrome in Children (MIS-C) [5-8] and adolescents associated with SARS-CoV-2, by the World Health Organization (WHO) [9].

Current study reports the case of a 15-year-old girl who presented with fever, raised inflammatory markers, showed multisystem involvement and echocardiographic changes were suggestive of post-COVID MIS.

\section{CASE REPORT}

A 15-year-old girl was presented with fever, rashes all over the body and redness of both eyes for 3 days. There was history of pain abdomen, multiple episodes of vomiting and loose motion, 4 days back. No significant past medical history was reported. There was family history of a relative being COVID positive 1 month ago; however, the child herself did not have any symptoms at that time. The following parameters were recorded on admission: Heart rate $=160 / \mathrm{min}$, blood pressure $=110 / 60 \mathrm{mmHg}$, respiratory rate $=30 / \mathrm{min}$, and $\mathrm{Temp}=103^{\circ} \mathrm{F}$. On examination, she was icteric with blanching erythematous rash all over the body, bilateral conjunctival congestion and hepatomegaly $(3 \mathrm{~cm})$. There were no meningeal signs. Baseline investigations done 2 days before admission showed mildly deranged liver function tests (LFT) (Serum bilirubin: $3.5 \mathrm{mg} / \mathrm{dl}$, Aspartate aminotransferase [AST]: $131 \mathrm{IU} / \mathrm{L}$, Alanine transaminase [ALT]: $145 \mathrm{IU} / \mathrm{L}$, Alkaline

Correspondence to: Dr. Meghmala Sadhukhan, Apollo Hospitals, Bhubaneswar, 251, Sainik School Road, Bhubaneswar - 751 005, Odisha, India. E-mail: meghmala123@yahoo.com

(C) 2021 Creative Commons Attribution-NonCommercial 4.0 International License (CC BY-NC-ND 4.0). 
phosphatase [ALP]: 247 IU/L) and raised C-reactive protein (16). Dengue serology and malaria antigen tests were also negative. Following admission, intravenous cefotaxime and amikacin were started empirically. Activated partial thromboplastin time and international normalized ratio was normal. Repeat LFT showed serum Bilirubin $2.9 \mathrm{mg} / \mathrm{dl}$ (conjugated $1.6 \mathrm{mg} / \mathrm{dl}$ and unconjugated $1.3 \mathrm{mg} / \mathrm{dl}$ ), albumin $3.1 \mathrm{mg} / \mathrm{dl}$, AST $67 \mathrm{IU} / \mathrm{L}$, ALT 84 IU/L, ALP 198 IU/L, Gamma-glutamyl transferase 174 IU/L, $\mathrm{Hb} 10.3 \mathrm{~g} / \mathrm{dl}$, and total white blood cell count $18,400 / \mu \mathrm{L}$. COVID TRUNAT, COVID IgG, scrub IgM, and leptospira serology were negative. Ultrasound abdomen showed mild hepatomegaly. Urine routine microscopy showed 6-8 pus cells/hpf but urine culture and blood culture were negative.

The child was continuing to have fever even after $48 \mathrm{~h}$, developed cough and on auscultation of the chest there was $\mathrm{B} / \mathrm{L}$ crepitations. Chest X-ray showed mild patchy shadows in both lung fields. High-resolution computed tomography chest showed bilateral patchy subsegmental consolidation in basal segment (CORAD score: 4). Two-dimensional echocardiogram showed dilated left main coronary artery $(3.4 \mathrm{~mm})$ suggestive of KD. Repeat CRP was 14.2 and ESR 98. Serum Ferritin $(342.9 \mathrm{ng} / \mathrm{mL})$, D-Dimer (721.309 ng/mL), and IL-6 $(29.55 \mathrm{pg} / \mathrm{ml})$ levels were also raised. Repeat COVID TRUNAT was negative.

The child was shifted to isolation ward and intravenous immunoglobulin (IVIG) was given at $2 \mathrm{~g} / \mathrm{kg}$ along with high dose of aspirin (100 mg/kg/day). In view of raised D-Dimer, low molecular weight heparin was also started at $40 \mathrm{mg}$ subcutaneous daily. The child became afebrile after $48 \mathrm{~h}$ and was clinically better. Repeat inflammatory markers were also improved. Therefore, she was discharged with prescription of low dose aspirin. Repeat echocardiogram after 4 weeks of discharge showed reduction in the diameter of the left main coronary artery to $3 \mathrm{~mm}$.

\section{DISCUSSION}

Some children with COVID-19 develop a cytokine storm syndrome which may require intensive care admission [1-3]. Children presenting with persistent fever, features of inflammation such as neutrophilia, high CRP, and lymphopenia, and single or multiorgan dysfunction were classified in the UK as "PMIS-TS" by the Royal College of Paediatrics (RCPCH) regardless of the SARSCoV-2 RT-PCR test results [2,3]. The similar hyperinflammatory condition was named as "MIS-C" by the Centre for Disease Control and Prevention [5-8]. The phenotypes under this category are typical/atypical KD, Kawasaki shock syndrome, toxic shock syndrome, and macrophage activation syndrome/hemophagocytic lymphohistiocytosis [5-8]. The pathophysiology is still not very clearly understood, but from the studies so far it is thought to be due a dysregulated hyperimmune response to earlier infection with the SARS-CoV-2 virus [5-8]. Most children are found to be RT-PCR negative during this stage but test positive to the antibodies (IgG) to the virus. Therapeutic options include IVIG, corticosteroids, tocilizumab, and anakinra from the evidence obtained from previously published reports [5-8].
Our case was under the atypical KD group both in terms of the age of presentation and incomplete clinical features which made the diagnosis confusing, initially. She also satisfied the WHO definition of COVID-19 associated MIS-C. The pathophysiology of cardiovascular involvement in COVID-19 is suggested to be due to endotheliitis, resulting from the virus triggering an inflammatory cascade in the endothelial cells of blood vessels [10]. In addition, certain host genetic and environmental factors have also being implicated [10].

In our case, both RT-PCR and serology (IgG) for SARS$\mathrm{CoV}-2$ were negative but there was a history of contact with a documented RT-PCR positive family member, about 1 month ago. From previously published data, it has been seen that the sensitivity of RT-PCR tests is low and it is affected by many external factors such as the sampling time, whether the sample was taken correctly and the performance of test kits [11]. Ai et al. reported that 413 of 1014 (41\%) RT-PCR test results of infected patients were negative at the initial presentation [12]. The advantage of serological tests over RT-PCR tests is that antibodies persist in the blood for longer time and they are structurally more stable during the sample processing steps $[11,12]$. However, serological tests could be negative in the infected patients at an early stage of infection $[11,12]$. In a literature review, serologic tests performed in a cohort of 155 patients, 130 were positive (83.8\%), suggesting that, the sensitivity of COVID-19 serology tests ranges from $72.7 \%$ to $100 \%[13]$.

\section{CONCLUSION}

COVID-19 associated MIS-C is a new clinical condition observed in children and adolescents during this pandemic. The patients with MIS-C may deteriorate quickly if not investigated and managed on time. Pediatricians therefore need to be aware of this condition during their day-to-day practice.

\section{REFERENCES}

1. Bialek S, Gierke R, Hughes M, McNamara LA, Pilishvili T, Skoff T. Coronavirus disease 2019 in children United States, February 12-April 2, 2020. MMWR Morb Mortal Wkly Rep 2020;69:422-6.

2. Choi SH, Kim HW, Kang JM. Epidemiology and clinical features of coronavirus disease 2019 in children. Clin Exp Pediatr 2020;63:125.

3. Toubiana J, Poirault C, Corsia A, Bajolle F, Fourgeaud J, Angoulvant F, et al. Kawasaki-like multisystem inflammatory syndrome in children during the covid-19 pandemic in Paris, France: Prospective observational study. BMJ 2020;369:m2094

4. Chang LY, Lu CY, Shao PL, Lee PI, Lin MT, Fan TY, et al. Viral infections associated with Kawasaki disease. J Formos Med Assoc 2014;113:148-54.

5. European Centre for Disease Prevention and Control. Paediatric Inflammatory Multisystem Syndrome and SARS-CoV-2 Infection in Children 15 May 2020. Stockholm: European Centre for Disease Prevention and Control; 2020.

6. Verdoni L, Mazza A, Gervasoni A. An outbreak of severe Kawasakilike disease at the Italian epicentre of the SARS-CoV-2 epidemic: An observational cohort study. Lancet 2020;395:1771-8.

7. Riphagen S, Gomez X, Gonzalez-Martinez C, Wilkinson N, Theocharis P. Hyperinflammatory shock in children during COVID-19 pandemic. Lancet 2020;395:1607-8.

8. Pouletty M, Borocco C, Ouldali N. Paediatric multisystem inflammatory syndrome temporally associated with SARS-CoV-2 mimicking Kawasaki 
disease (Kawa-COVID-19): A multicentre cohort. Ann Rheum Dis 2020;79:999-1006.

9. World Health Organization. Multisystem Inflammatory Syndrome in Children and Adolescents with COVID-19. Geneva: World Health Organization; 2020. Available from: https://www.who.int/publications/i/ item/multisystem-inflammatory-syndrome-in-children-and-adolescentswith-covid-19. [Last accessed on 2020 May 15].

10. Varga Z, Flammer AJ, Steiger P. Endothelial cell infection and endotheliitis in COVID-19. Lancet 2020;395:1417-8.

11. Younes N, Al-Sadeq DW, Al-Jighefee H. Challenges in laboratory diagnosis of the novel coronavirus SARS-CoV-2. Viruses 2020;12:582.

12. Ai T, Yang Z, Hou H. Correlation of chest CT and RTPCR testing in coronavirus disease 2019 (COVID-19) in China: A report of 1014 cases.
Radiology 2020;296:E32-40.

13. Paul SW, Robert SP, Mahan G, José L. Estimating the false-negative test probability of SARS-CoV-2 by RT-PCR. Euro Surveill 2020;25:2000568.

Funding: None; Conflicts of Interest: None Stated.

How to cite this article: Sadhukhan M, Joshi RK, Pahi PP. Multisystem Inflammatory Syndrome in an adolescent girl presenting as atypical Kawasaki-like illness associated with coronavirus disease-19. Indian J Case Health. 2021; 8(8):302-304. 